\title{
Heat shock protein 27 interacts with vimentin and prevents insolubilization of vimentin subunits induced by cadmium
}

\author{
Jae-Seon Lee ${ }^{1,2 *}$, Mei-Hua Zhang ${ }^{1 *}$, \\ Eun Kyung Yun ${ }^{1}$, Dongho Geum ${ }^{3}$, \\ Kyungjin $\mathrm{Kim}^{3}$, Tae-Hyung Kim ${ }^{1}$, \\ Yun-Sook Lim $^{1}$ and Jeong-Sun Seo ${ }^{1,4}$ \\ ${ }^{1}$ ILCHUN Molecular Medicine Institute MRC \\ Department of Biochemistry and Molecular Biology \\ Seoul National University College of Medicine \\ Seoul 110-799, Korea \\ ${ }^{2}$ Laboratory of Functional Genomics \\ Korea Institute of Radiological \& Medical Sciences \\ Seoul 139-706, Korea \\ ${ }^{3}$ School of Biological Sciences \\ Seoul National University \\ Seoul 151-742, Korea \\ ${ }^{4}$ Corresponding author: Tel, 82-2-740-8246; \\ Fax, 82-2-741-5423; E-mail, jeongsun@snu.ac.kr \\ ${ }^{*}$ These authors contributed equally to this work.
}

Accepted 26 August 2005

Abbreviation: ERK, extracellular signal-regulated kinase; GST, glutathione S-transferase; HSP, heat-shock protein; JNK, Jun $N$-terminal kinase; MAPK, mitogen-activated protein kinase

\begin{abstract}
Vimentin is an intermediate filament that regulates cell attachment and subcellular organization. In this study, vimentin filaments were morphologically altered, and its soluble subunits were rapidly reduced via cadmium chloride treatment. Cadmium chloride stimulated three major mitogen-activated protein kinases (MAPKs): extracellular signal-re gulated kinase (ERK), C-Jun N-terminal kinase (JNK), and $\mathrm{p} 38$, and led apoptotic pathway via caspase-9 and caspase-3 activations. In order to determine whether MAPKs were involved in this cadmium-induced soluble vimentin disappearance, we applied MAPKspecific inhibitors (PD98059, SP600125, SB203580). These inhibitors did not abolish the cadmium-induced soluble vimentin disappearance. Caspase and proteosome degradation pathway were also not involved in soluble vimentin disappearance. When we observed vimentin levels in soluble and insoluble fractions, soluble vimentin subunits shifted to an insoluble fraction. As we discovered that heat-
\end{abstract}

shock protein 27 (HSP27) was colocalized and physically associated with vimentin in unstressed cells, the roles of HSP27 with regard to vimentin were assessed. HSP27-overexpressing cells prevented morphological alterations of the vimentin filaments, as well as reductions of soluble vimentin, in the cadmium-treated cells. Moreover, HSP27 antisense oligonucleotide augmented these cadmium-induced changes in vimentin. These findings indicate that HSP27 prevents disruption of the vimentin intermediate filament networks and soluble vimentin disappearance, by virtue of its physical interaction with vimentin in cadmium-treated SK-N-SH cells.

Keywords: apoptosis; cadmium chloride; heat shock protein; mitogen activated protein kinases; molecular chaperones; vimentin

\section{Introduction}

Vimentin is a major component of intermediate filament polypeptide, and forms a highly insoluble network in the fibroblasts. Vimentin surrounds the nucleus, extending outwards to the cell periphery. It is responsible for cell elongation, regulation of cell attachment, subcellular organization, and signal transduction from the plasma membrane to the nucleus (Owen et al., 1996). Vimentin undergoes proteolysis, and corresponds to ubiquitinated polypeptides formed during apoptosis (Fujita et al., 2003).

Cadmium, a toxic heavy metal, is quite harmful to human beings, due primarily to its long biological half-life. Cadmium is a ubiquitous environmental contaminant, and exerts mutagenic, carcinogenic, and teratogenic effects. Humans are exposed to cadmium in its forms as an industrial pollutant, a food contaminant, and as a component of tobacco smoke (Li et al., 2000b). Cadmium-induced DNA damage may be the underlying cause of carcinogenic activity, along with cadmium-related effects on oncogene expression (Wang and Templeton, 1998). Cadmium can induce apoptosis and stress response in a variety of tissues and cells (Galan et al., 2000). The nervous system is a major target for the cadmium toxicity (Yoshida, 2001). It has been reported that cadmium disturbs important nervous system functions (Hart et al., 1989). However, the mechanisms underlying many of the activities and effects of cadmium remain poorly understood. 
Heat-shock proteins (HSPs) play vital roles as molecular chaperones in the folding, transport, and assembly of newly synthesized polypeptides. HSPs are induced in response to a wide range of chemicals and biological signals, including heat shock, heavy metals, UV- and $\gamma$-radiation, oxidative stress, viral and bacterial infections, and osmotic stress (Morimoto et al., 1990; Lee and Seo, 2002). This suggests that HSPs may be involved in a general cellular defense mechanism. They contribute to cellular protection via the restoration of denatured proteins, or via physical interaction with specific target proteins (Li et al., 2000a; Park et al., 2001). HSPs are highly conserved from bacteria to mammals, and are generally divided into five major classes, according to molecular size: HSP27, HSP60, HSP70, HSP90, and HSP110. HSP27 exhibits an ATP-independent chaperone activity which prevents protein aggregation, and function as anti-apoptotic molecule (Jakob et al., 1993; Ehrnsperger et al., 1997; Paul et al., 2002). Several mechanisms have been proposed to account for the anti-apoptotic activities of HSP27 (Bruey et al., 2000). The cytoprotective effect of HSP27 has been attributed to its function as a modulator of the integrity of F-actin microfilaments (Gerthoffer and Gunst, 2001). Small HSPs, HSP27 and $\alpha B$-crystallin, are associated specifically with intermediate filaments in human pathologies involving intermediate filament aggregates (Perng et al., 1999). HSP27-overexpressing cells showed resistance to cell death by cadmium chloride (Wu and Welsh, 1996). A potential role for HSP27 in the cellular response to cadmium was suggested due to cadmium-dependent increase in HSP27 (Bonham et al., 2003).

In this study, we demonstrated that HSP27 is a binding partner of vimentin, by conducting a co-immunoprecipitation assay on human neuroblastoma SK-N-SH cells. In order to ascertain the role of HSP27 with regard to vimentin, we observed alterations in vimentin during cadmium-induced apoptosis, using HSP27-antisense or HSP27-overexpression strategies. Our results indicate that HSP27 plays an important role in preventing the alteration of vimentin filaments and vimentin subunit levels in the cadmium-induced apoptotic pathway.

\section{Materials and Methods}

\section{Cell culture and reagents}

SK-N-SH cells were maintained at $37^{\circ} \mathrm{C}$ in DMEM medium supplemented with $10 \%$ heat-inactivated fetal bovine serum (GIBCO, Carlsbad, CA) in a humidified $95 \%$ air, $5 \% \mathrm{CO}_{2}$ incubator. $\mathrm{CdCl}_{2}$ was purchased from Sigma (St. Louis, MO). Antibodies against JNK, ERK, p38, phospho-JNK (Thr183/Tyr185), phos-
pho-ERK (Thr202/yr204), and phospho-p38 (Thr180/ Tyr182) were obtained from Cell Signaling (Beverly, MA). Anti-HSP27, anti-actin, anti-caspase-3, anti-vimentin, and peroxidase-conjugated secondary antibodies were purchased from Santa Cruz Biotechnology (Santa Cruz, CA). Anti-caspase-9 antibody was obtained from BD Pharmigen (San Jose, CA). MG132, ALLN, PD98059, and SB203580 were purchased from Calbiochem (San Diego, CA). SP600125 was obtained from TOCRIS (Bristol, UK). Enhanced chemiluminescense $(E C L)$ western blotting detection reagents were obtained from Amersham (Little Chalfont).

\section{Western blot analysis}

After treatment of cadmium chloride, cells were washed with cold PBS three times and collected with cell scraper. Protein extracts were prepared as described in Lee and Seo (2002). Extracts were subjected to SDS-PAGE electrophoresis and transferred to a nitrocellulose membrane. The blot was blocked with Tris-buffered saline (TBS) $(20 \mathrm{mM}$ Tris, $\mathrm{pH} 7.5,137 \mathrm{mM} \mathrm{NaCl}$ ) containing $5 \%$ nonfat milk for $1 \mathrm{~h}$ at room temperature, and incubated with primary antibodies (1:1,000 dilutions) in TBST (20 mM Tris, $\mathrm{pH} 7.5,137 \mathrm{mM} \mathrm{NaCl}, 0.2 \%$ Tween 20) containing $3 \%$ nonfat dry milk for $2 \mathrm{~h}$ at room temperature. The blots were washed three times with TBST, and incubated with horseradish peroxidase-conjugated secondary antibodies (1:5,000 dilutions) in TBST containing $3 \%$ nonfat dry milk for $2 \mathrm{~h}$ at room temperature. After washing three times with TBST, the blots were developed with the ECL detection system. Soluble and insoluble fractions were prepared as described in Yaglom et al. (2003).

\section{Co-immunoprecipitation}

SK-N-SH cells were incubated with lysis buffer (50 $\mathrm{mM}$ Tris- $\mathrm{HCl}, \mathrm{pH} 7.5,1 \%$ Nonidet P-40, $150 \mathrm{mM}$ $\mathrm{NaCl}, 0.5 \%$ sodium deoxycholate, and protease inhibitors). The lysates $(500 \mu \mathrm{g})$ were precleared by gentle agitation with protein $\mathrm{A} / \mathrm{G}$-agarose beads at $4^{\circ} \mathrm{C}$ for $4 \mathrm{~h}$. And then $2 \mu \mathrm{g}$ anti-vimentin (V9) and anti-HSP27 antibodies were added and incubated for $4 \mathrm{~h}$ at $4^{\circ} \mathrm{C}$. The immunocomplexes were collected on protein $A / G$-agarose beads at $4^{\circ} \mathrm{C}$ overnight. After washing three times with lysis buffer, they were analyzed by Western blotting with antivimentin and anti-HSP27 antibodies. In a pull-down binding assay, SK-N-SH cell extracts $(1 \mathrm{mg})$ were incubated with glutathione S-transferase (GST)-vimentin immobilized on glutathione-sepharose 4B beads at $4^{\circ} \mathrm{C}$ for $4 \mathrm{~h}$. After centrifugation, the beads were washed three times, and then the bound complex was analyzed by immunoblot using antiHSP27 or anti-vimentin antibodies. 


\section{Immunofluorescence staining}

Cells grown on coverslips were gently rinsed with PBS, and fixed in $3.7 \%$ paraformaldehyde in PBS for 15 minutes at room temperature. After washing with PBS, they were permeabilized with $0.1 \%$ Triton $X$ 100 in PBS for $10 \mathrm{~min}$ at room temperature. Nonspecific protein absorption was inhibited by incubating cells for $1 \mathrm{~h}$ in PBS containing 1\% BSA. For immunostaining of HSP27 or vimentin, cells were incubated with anti-HSP27 or anti-vimentin antibodies for $2 \mathrm{~h}$ at room temperature. After washing three times with PBST, they were incubated for 2 hour with FITC-conjugated or Texas red-conjugated secondary antibodies and washed three times with PBST. The immunolabeled cells were examined with laser scanning confocal microscope (Bio-Rad, MRC 1024).

\section{Transfection experiments}

SK-N-SH cells were grown to $70 \%$ confluence. Transfection was carried out using Effectene reagent (Qiagen, Valencia) with pRetroTet-On or pRetroTetOn-hsp27 plasmids (Geum et al., 2002) according to the manufacturer's protocol. Cells were transfected with $10 \mu \mathrm{M}$ HSP27 sense or anti-sense oligonucleotides before treatment with cadmium chloride. Sense (5'-GCATTCCGTGCTATGATT-3') and anti-sense (5'-AATCATAGCACGGAATGC-3') oligonucleotides of HSP27 were prepared as described in Lee et al. (2001).

\section{Results}

\section{Vimentin intermediate filaments and soluble vimentin subunits are affected by cadmium}

We assessed the level of soluble vimentin subunits in cadmium-treated SK-N-SH cells. Vimentin levels were found to decrease precipitously in a dose- and time-dependent manner in soluble fractions, at concentrations of $0.2,0.5$, and $1 \mathrm{mM}$ cadmium (Figure $1 \mathrm{~A})$. High concentrations of cadmium $(1 \mathrm{mM})$ resulted in the rapid disappearance of soluble vimentin, within $30 \mathrm{~min}$ (Figure 1B). In order to visualize the morphological changes taking place in the vimentin filaments at this concentration, we treated the SK-N$\mathrm{SH}$ cells with $1 \mathrm{mM}$ cadmium, and examined the vimentin filaments under immunofluorescence confocal microscopy (Figure 1C). In unstressed SK-N-

A

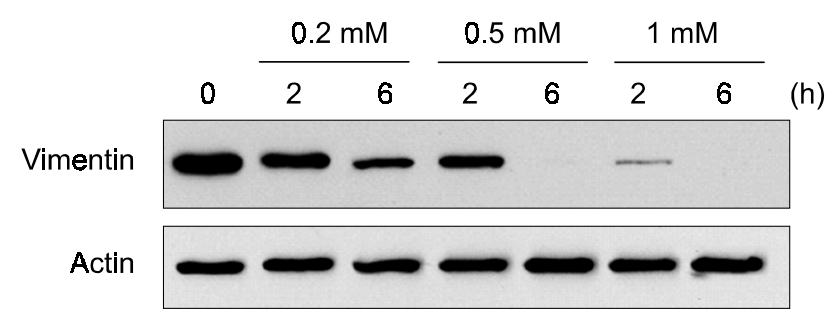

B

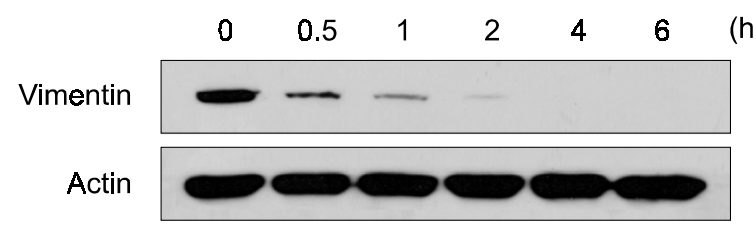

C

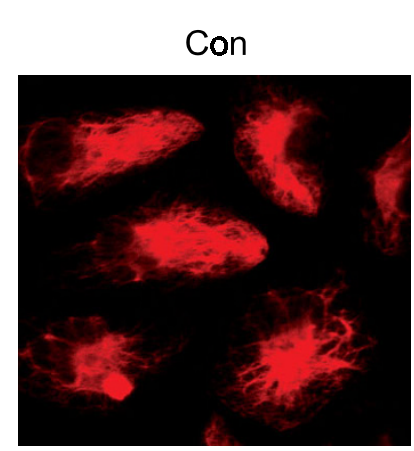

D

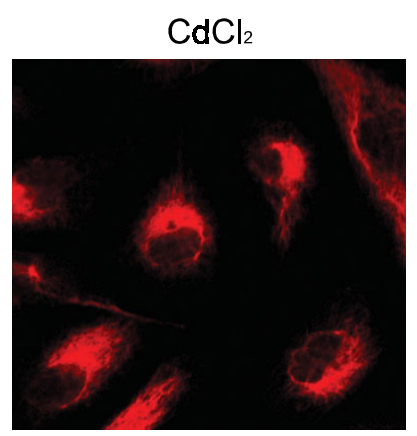

Figure 1. Changes in soluble vimentin level and vimentin organization in cadmium-treated SK-N-SH cells. (A) Decreased level of soluble vimentin due to cadmium exposure. Lysates from cadmiumtreated SK-N-SH cells were subjected to immunoblot analysis with anti-vimentin antibody. Soluble vimentin subunits were decreased in dose- and time-dependent manners. (B) The rapid disappearance of soluble vimentin in $1 \mathrm{mM}$ cadmium chloride. (C) Cadmium-induced morphological changes of the vimentin filaments. SK-N-SH cells were untreated or treated with $1 \mathrm{mM}$ cadmium chloride for $1 \mathrm{~h}$. Cells were subjected to indirect immunofluorescence staining, using anti-vimentin as a primary antibody and FITC-conjugated as secondary antibody. 
SH cells, vimentin filaments surrounded the perinuclear region, and extended in a fine network into the cytoplasm. In cadmium-treated SK-N-SH cells, vimentin filaments collapsed around the nuclear region with a loss of the fine cytoplasmic networks.

\section{Cadmium-induced activation of mitogen activated protein kinases and Caspase-3 are not involved in soluble vimentin disappearance}

Cadmium has been reported to induce apoptosis and stress response. Caspase- 9 and caspase- 3 in SK-N-SH cells were activated by treatment with 1 mM cadmium (Figure 2A). However, soluble vimentin levels had clearly decreased prior to the activation of caspase- 9 and caspase- 3 . This suggests that the cadmium-induced apoptotic pathway is mediated via the mitochondria-dependent pathway. However, soluble vimentin disappearance did not correlate well with caspase-3 activation. We attempted to determine whether the phosphorylation of mitogen-activated protein kinases (MAPKs) were involved in this rapid disappearance of soluble vi- mentin. Three major MAPKs, extracellular signal-regulated kinase (ERK), Jun N-terminal kinase (JNK), and p38, were rapidly phosphorylated (within 30 minutes) by cadmium treatment (Figure 2A). To clarify the role of MAPKs in the disappearance of soluble vimentin, MAPK inhibitors were added prior to cadmium treatment. The MAPK-specific inhibitors, ERK inhibitor (PD98059), JNK inhibitor (SP600125), and p38 inhibitor (SB203580), did not prevent the disappearance of soluble vimentin (Figure $2 \mathrm{~B}$ ). When we detected levels of HSPs, i.e., HSP90, HSP70, and HSP27, there was no detectable change in HSP90, HSP70, and HSP27 levels within $6 \mathrm{~h}$ after cadmium treatment (data not shown). The proteasome-mediated degradation pathway has been implicated in the proteolysis of a variety of substrates (Welch, 1992; Morishima, 1999), and also has multiple targets during apoptosis (Wojcik, 2002). In order to ascertain whether proteasome-mediated pathways are directly involved in cadmium-induced soluble vimentin disappearance, we assessed the effects of proteasome inhibitors. As shown in Figure $3 \mathrm{~A}$, the proteasome inhibitor MG132 had no effect
A

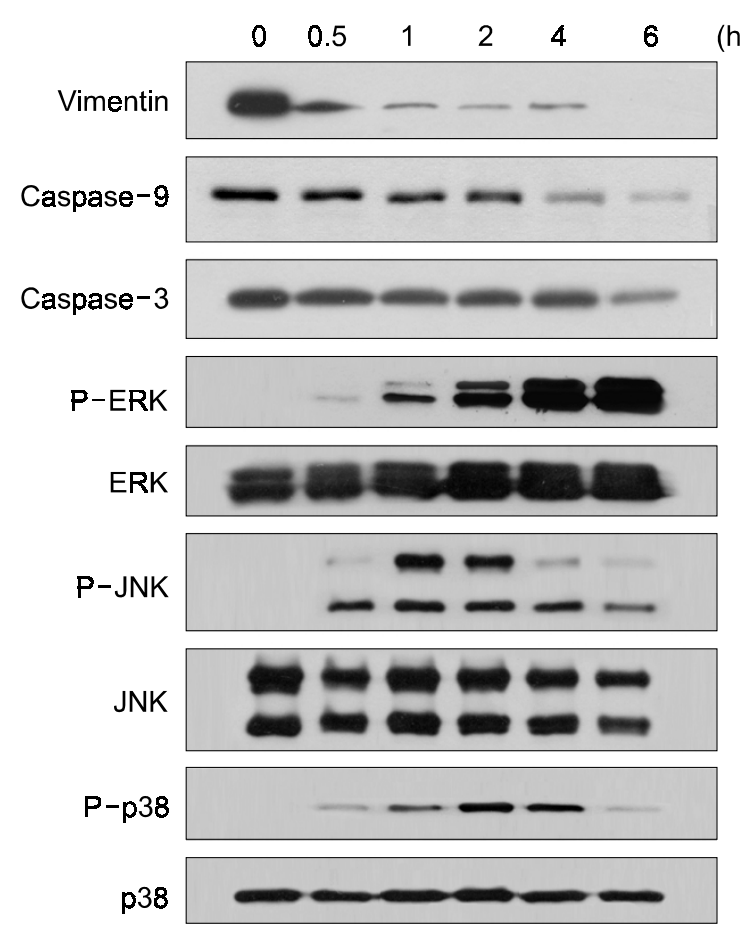

B

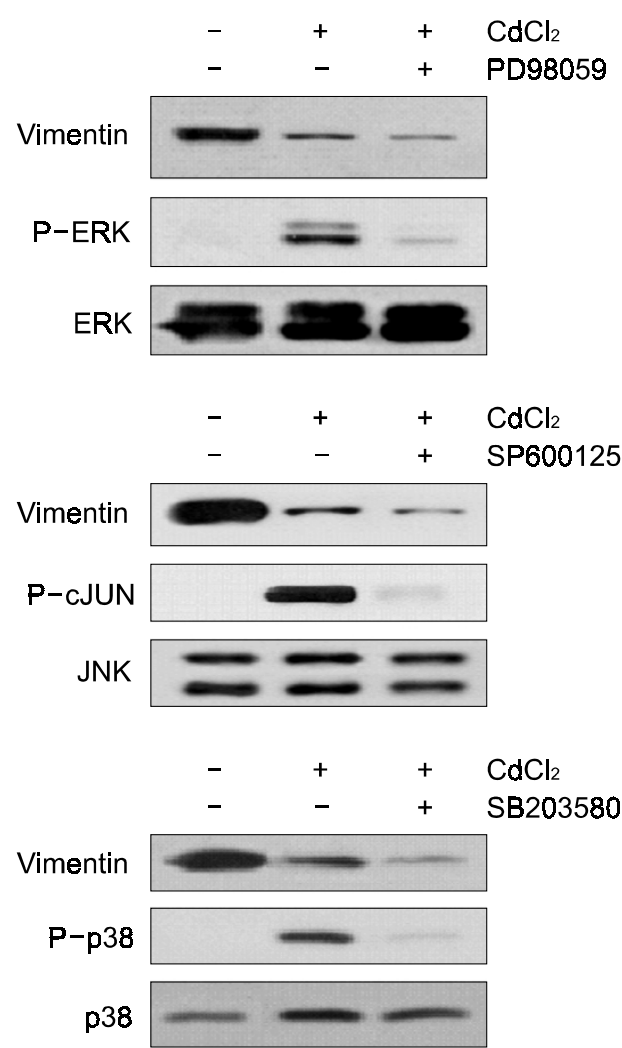

Figure 2. Changes in soluble vimentin level were not attributable to the activation of caspase- 3 and MAPKs. (A) Cadmium treatment induced cleavage of caspases and activation of MAPKs. SK-N-SH cells were treated with $1 \mathrm{mM}$ cadmium chloride for indicated periods of time, and then assessed by immunoblot analysis. (B) Effect of MAPK inhibitors on soluble vimentin disappearance. SK-N-SH cells were preincubated, either with or without PD98059 $(25 \mu \mathrm{M})$, SP600125 $(25 \mu \mathrm{M})$, and SB203580 $(20 \mu \mathrm{M})$ for $1 \mathrm{~h}$, and then treated with $1 \mathrm{mM}$ cadmium chloride for $1 \mathrm{~h}$. Cell lysates were analyzed via immunoblotting. 
A

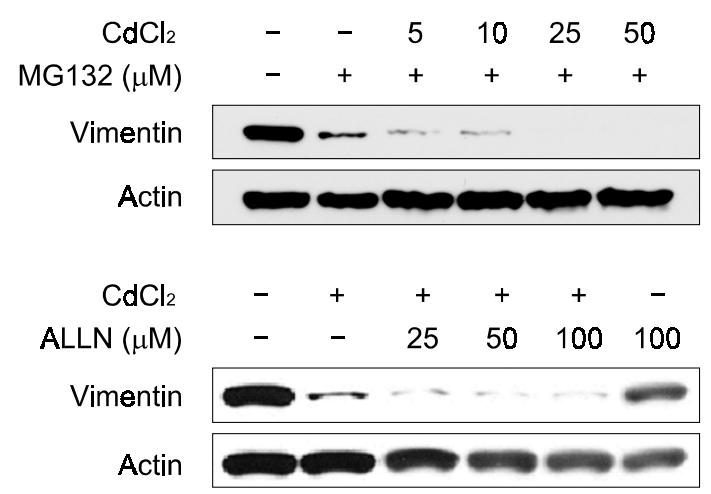

B
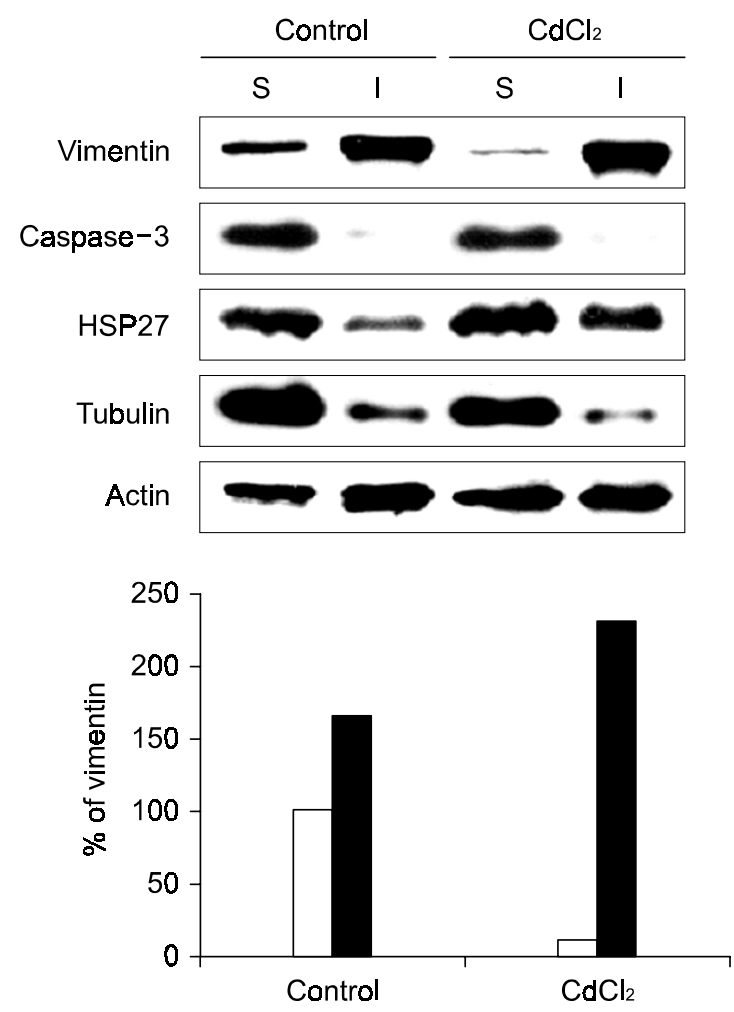

Figure 3. Soluble vimentin shifted into insoluble fraction due to cadmium exposure. (A) Effects of proteasome and calpain inhibitors on soluble vimentin disappearance. SK-N-SH cells were preincubated with MG-132 or ALLN for $1 \mathrm{~h}$, then treated with $1 \mathrm{mM}$ cadmium chloride for 1 $\mathrm{h}$. Immunoblot analyses were carried out with anti-vimentin and anti-actin antibodies. (B) Changes of vimentin level in soluble and insoluble fractions. Vimentin and HSP27 levels were examined in soluble and insoluble fractions, which were prepared with untreated and cadmium-treated SK-N-SH cells. Graph shows the mean of two representative studies.

on vimentin disappearance. As cadmium-induced apoptosis was mediated through a calpain-dependent pathway (Li et al., 2000), we attempted next to discern whether the disappearance of soluble vi- mentin might be inhibited by a calpain inhibitor. Soluble vimentin disappearance was not affected by the calpain inhibitor, ALLN (Figure 3A).

\section{Cadmium redistributes soluble vimentin subunits to insoluble fraction}

As soluble vimentin disappearance had proven independent of caspase, proteasome, and calpain, we surmised that soluble vimentin might aggregate or bind to large structures, thereby becoming insoluble. To test this possibility, cell lysates were fractionated into soluble and insoluble fractions, and immunoblotted with anti-vimentin antibody. Upon cadmium treatment, soluble vimentin shifted into an insoluble fraction (Figure 3B). HSP27, to some degree, also shifted into an insoluble fraction. However, caspase3 remained in the soluble fraction. The distribution of tubulin and actin remained unaffected by cadmium. This suggests that cadmium specifically redistributes soluble vimentin subunits.

\section{HSP27 associates physically with vimentin}

Previous studies have pointed to an interaction between the intermediate filament proteins and the chaperone proteins (Evans, 1998; Perng et al., 1999). Also, some amount of HSP27 shifted into an insoluble fraction along with the vimentin (Figure 3B). In order to characterize the possible association between HSP27 and vimentin, the intracellular localization of HSP27 and vimentin was analyzed via confocal microscopy. Vimentin was localized in the cytoplasm with strong perinuclear staining, and HSP27 was predominantly cytoplasmic in the SK-N$\mathrm{SH}$ cells. When the staining images of HSP27 and vimentin were merged, they were found to coincide both in the cytosol and in the perinuclear region (Figure 4A). In order to test whether HSP27 physically binds to vimentin, we carried out in vitro pull-down assay. Glutathione S-transferase (GST)vimentin was pulled out HSP27 from SK-N-SH cell extracts as shown in Figure 4B. Co-immunoprecipitation analyses were also performed, with either anti-vimentin or anti-HSP27 antibodies. When immunoprecipitated pellets were analyzed using antivimentin or anti-HSP27 antibodies, HSP27 physically interacted with vimentin in vivo (Figure 4C). These experiments indicate that vimentin exists in a complex with HSP27 in intact cells.

\section{HSP27 prevents insolubilization of vimentin subunits and morphological changes of vimentin filaments}

Since HSP27 associates physically with vimentin, we attempted to determine whether HSP27 prevents 
A

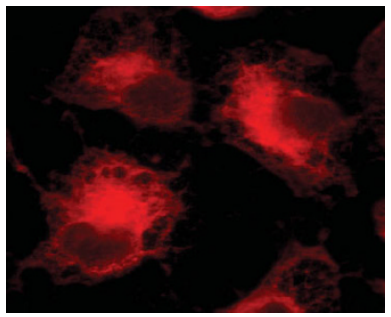

Vimentin

B

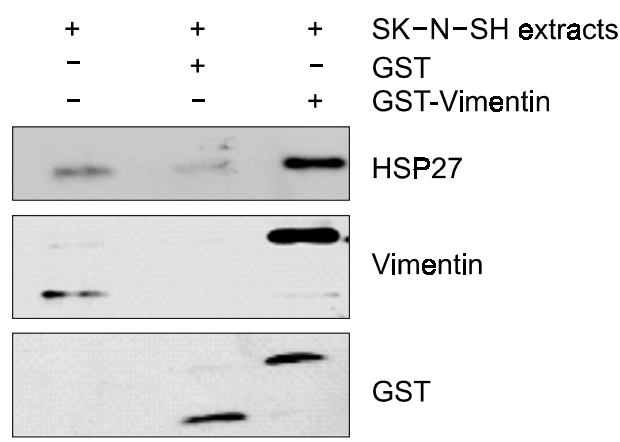

the disappearance of soluble vimentin subunits. Vimentin disappearance in the soluble fraction was examined after transfection with pRetroTet-On (empty vector) or pRetroTet-On-hsp27 (hsp27-overexpressing vector). In HSP27-overepressing cells, vimentin disappearance was reduced significantly, and the vimentin filaments were protected from collapse, as compared to the empty vector-transfected cells (Figure 5A). We also conducted HSP27 anti-sense oligonucleotide transfection. When cells were transfected with HSP27 anti-sense oligonucleotides prior to cadmium exposure, vimentin disappearance accelerated, to a rate consistent with HSP27 sense oligonucleotide-transfected cells (Figure 5B). In addition, the fine fibrillar vimentin network collapsed further, concentrating around the nucleus to a higher extent than in the sense oligonucletide-treated cells in response to cadmium chloride (Figure 5B). HSP27 levels in cells transfected with either sense or antisense HSP27 oligonucleotides were determined by Western blotting (Figure 5B). These findings suggest that HSP27 protects against the disappearance of soluble vimentin subunits and the disruption of vimentin filaments, which result from the cytotoxic effects of high concentrations of cadmium chloride.

\section{Discussion}

Small heat shock proteins participate in a variety of

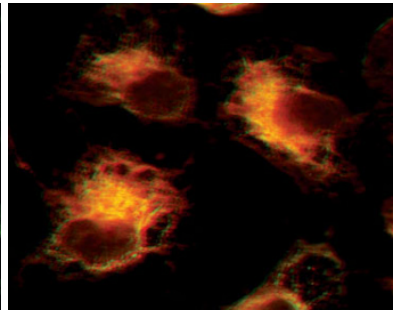

Merge

WB:

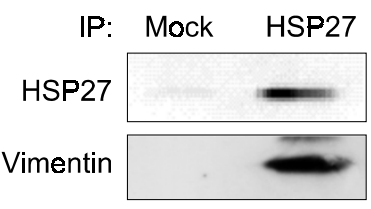

IP: Mock Vimentin

WB:

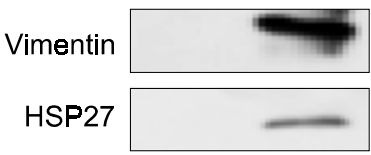

Figure 4. HSP27 interacts with vimentin both in vivo and in vitro. (A) Subcellular colocalization of HSP27 with vimentin filaments in the SK-N$\mathrm{SH}$ cells. SK-N-SH cells were costained with anti-vimentin and anti-HSP27 antibodies, and the images were obtained by confocal microscopy. (B) Association of vimentin with HSP27. SK-N-SH cell lysates were incubated with GST-vimentin immobilized on glutathione-sepharose $4 \mathrm{~B}$ beads at $4^{\circ} \mathrm{C}$ for $4 \mathrm{~h}$. After washing and elution of proteins from the beads, the bound proteins were analyzed by immunoblotting using anti-HSP27 and anti-vimentin antibodies. (C) Co-immunoprecipitation of HSP27 with vimentin. Cell lysates from SK-N-SH cells were immunoprecipitated using anti-vimentin or anti-HSP27 antibodies. The resulting pellets were subjected to SDS-PAGE, and analyzed by immunoblotting with anti-HSP27 and anti-vimentin antibodies.

cellular processes, including the suppression of protein aggregation, dynamics of cytoskeletal proteins, and cell growth and differentiation (Perng et al., 1999). HSP27, a member of the small heat shock proteins, is a powerful ATP-independent chaperone. HSP27 levels are generally low in unstressed cells. During stress response, HSP27 levels increased dramatically, and HSP27 becomes phosphorylated on its serine residues, assuming a cytoprotective role. Recent studies have demonstrated that HSP27 regulates apoptosis via several different mechanisms. HSP27 can maintain both redox homeostasis and mitochondrial stability (Concannon et al., 2003). HSP27 interacts with cytochrome $c$ to prevent the correct function of the apotosomes, and also interacts with procaspase- 3 to inhibit caspase- 3 activation (Concannon et al., 2003).

Intermediate filaments play a role in cell integrity, mobility, and differentiation (Hernandez, 2000; Salvador-Silva et al., 2001). Intermediate filaments are composed of lamins, keratins, and vimentins in a highly insoluble network. As vimentin filaments are responsible for cell attachment, it is important to maintain their integrity to preserve the attachment to the matrix (Prasad et al., 1998). Proteolytic degradation of vimentin was reported to be a component of the executive process of apoptosis (Hashimoto et al., 1998; Fujita et al., 2003).

Cadmium is a ubiquitous environmental toxicant, and controls cell growth and apoptosis through the 
A
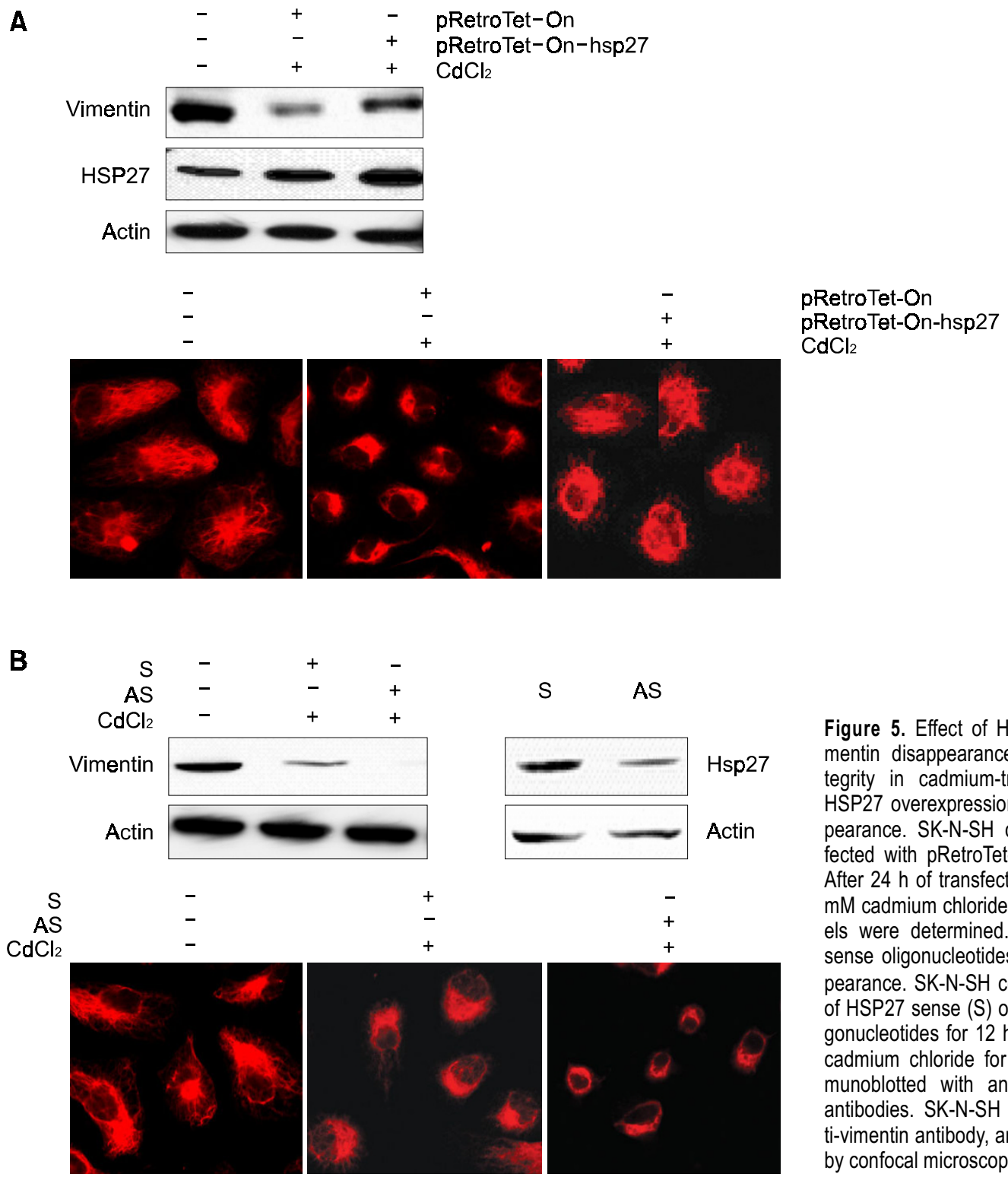

$S$

AS
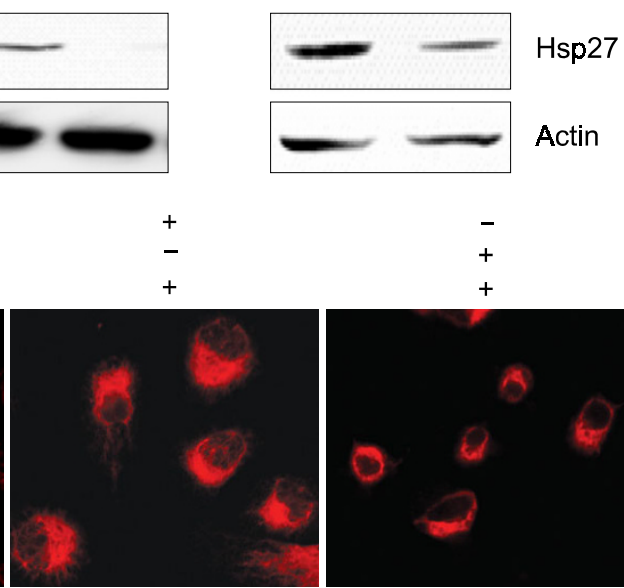

Figure 5. Effect of HSP27 per se on soluble vimentin disappearance and vimentin filament integrity in cadmium-treated cells. (A) Effect of HSP27 overexpression on soluble vimentin disappearance. SK-N-SH cells were transiently transfected with pRetroTet-On or pRetroTet-On-hsp27. After $24 \mathrm{~h}$ of transfection, they were exposed to 1 $\mathrm{mM}$ cadmium chloride for 1 hour, and vimentin levels were determined. (B) Effect of HSP27 antisense oligonucleotides on soluble vimentin disappearance. SK-N-SH cells were exposed to $10 \mu \mathrm{M}$ of HSP27 sense (S) or HSP27 anti-sense (AS) oligonucleotides for $12 \mathrm{~h}$, prior to exposure to $1 \mathrm{mM}$ cadmium chloride for $1 \mathrm{~h}$. Cell lysates were immunoblotted with anti-vimentin and anti-HSP27 antibodies. SK-N-SH cells were stained with anti-vimentin antibody, and the images were obtained by confocal microscopy. regulation of mitogen-activated protein kinases (MAPKs) (Galan et al., 2000). Three major MAPKs, ERK, JNK, and p38, are differentially activated, depending on the cadmium concentration (Chuang et al., 2000). The cadmium-induced apoptotic pathway has been reported to be caspase-independent (Shih et al., 2003; Shih et al., 2004). Galan et al., (2000), however, reported that cadmium-induced apoptosis was inhibited by the caspase inhibitors Z-VAD and DEVD-CHO. The signaling pathway and the role of MAPKs in cadmium-induced apoptosis require precise determination. Which MAPK has a key role and which caspase is involved in the cadmium-induced apoptotic pathway? We addressed this issue by observing the activation of MAPKs and caspases in the process of cadmium-mediated apoptosis. ERK, JNK, and p38 were evidently phosphorylated as a result of cadmium treatment. However, our results indicated that MAPKs were not involved in cadmium-induced vimentin disappearance. Although caspase- 3 has been tentatively implicated in the proteolytic digestion of vimentin (Hashimoto et al., 1998; Fujita et al., 2003), vimentin disappearance in the soluble fraction, induced by high concentrations ( $1 \mathrm{mM})$ of cadmium, was not mediated via any caspase-3-dependent pathway.

Small heat shock proteins interact with both microfilaments and intermediate filaments, facilitating the control of interactions between filaments in cellular networks (Perng et al., 1999). HSP27 is phosphor- 
ylated by MAPKAP kinase, downstream of p38 MAPK in the stress response pathway (Guay et al., 1997). HSP27 is, thus, involved in the regulation of microfilament dynamics, modifying the actin response to physiological agonists, and stabilizing the actin cytoskeleton under stress conditions (Guay et al., 1997). HSP27 and $\kappa B-c r y s t a l l i n$ colocalize with intermediate filament, glial fibrillary acidic protein (GFAP) in the astrocytoma cells (Perng et al., 1999). However, the association of small heat shock proteins with the soluble pool of GFAP did not prevent GFAP filament collapse in stressed cells. They therefore proposed that small heat shock proteins help manage interactions between filaments in their cellular networks.

In this study, we discovered that HSP27 is a novel binding partner of vimentin. HSP27 prevents the rapid insolubilization of soluble vimentin induced by high concentrations of cadmium exposure, by binding directly to vimentin. HSP70 has been suggested to facilitate the reassembly of focal adhesions by increasing paxillin re-solubilization (Mao et al., 2004). Hsp70 prevented the translocation of paxillin into the detergent-insoluble fraction during ATP depletion. We demonstrated that HSP27, like HSP70, associates with vimentin and ameliorates vimentin insolubilization and vimentin filament collapse. Our observations suggest that the interaction of HSP27 with the intermediate filaments may help to maintain the structure and integrity of vimentin filaments under stress conditions, due to maintenance of the soluble vimentin subunit pool. As the assembly of intermediate filaments is a very active process (Söllner et al., 1985), the maintenance of a soluble vimentin pool also maintains the integrity of vimentin filaments in cellular networks. This event may be important with regard to cell survival. Our results were supported by the report of a mutation in a small heat shock protein, which induces intermediate filament aggregation in muscles (Vicart et al., 1998). Our findings may aid in the understanding of the molecular mechanisms underlying HSP27's role as a major cytoprotective molecule via the inhibition of alterations in vimentin, ultimately maintaining cell attachment and integrity.

\section{Acknowledgement}

We thank Drs. M. Munkhzol, Amarjargal Olzvoi and $B-G$ Jang for their help in this study.

\section{References}

Bonham RT, Fine MR, Pollock FM, Shelden EA. Hsp27, Hsp70, and metallothionein in MDCK and LLC-PK1 renal epithelial cells: effects of prolonged exposure to cadmium.
Toxicol Appl Pharmacol 2003;191:63-73

Bruey JM, Ducasse C, Bonniaud P, Ravagnan L, Susin SA, Diaz-latoud C, Gurbuxani, S, Arrigo AP, Kroemer G, Solary E, Garrido C. Hsp27 negatively regulates cell death by interacting with cytochrome c. Nature Cell Biol 2000;2: $645-52$

Chuang SM, Wang IC, Yang JL. Roles of JNK, p38 and ERK mitogen-activated protein kinases in the growth inhibition and apoptosis induced by cadmium. Carcinogenesis 2000; 21:1423-32

Concannon CG, Gorman AM, Samali A. On the role of Hsp27 in regulating apoptosis. Apoptosis 2003;8:61-70

Ehrnsperger M, Graber S, Gaestel M, Buchner J. Binding of non-native protein to Hsp25 during heat shock creates a reservoir of folding intermediates for reactivation. EMBO J 1997;16:221-9

Evans RM. Vimentin: the conundrum of the intermediate filament gene family. BioEssays 1998;20:79-86

Fujita J, Bandoh S, Yang Y, Wu F, Ohtsuki Y, Yoshinouchi T, Ishida T. High molecular weight vimentin complex is formed after proteolytic digestion of vimentin by caspase-3: detection by sera of patients with interstitial pneumonia. Microbiol Immunol 2003;47:447-51

Galan A, Garcia-Bermejo, ML, Troyano A, Vilaboa NE, de Blas E, Kazanietz MG, Aller P. Stimulation of p38 mitogen-activated protein kinase is an early regulatory event for the cadmium-induced apoptosis in human promonocytic cells. J Biol Chem 2000;275:11418-24

Gerthoffer WT, Gunst SJ. Invited review: focal adhesion and small heat shock proteins in the regulation of action remodeling and contractility in smooth muscle. J Appl Physiol 2001;91:963-72

Geum D, Son GH, Kim K. Phosphorylation-dependent celIular localization and thermoprotective role of heat shock protein 25 in hippocampal progenitor cells. J Biol Chem 2002;277:19913-21

Guay J, Lambert H, Gingras-Breton G, Lavoie JN, Huot J, Landry J. Regulation of actin filament dynamics by p38 MAP kinase-mediated phosphorylation of heat shock protein 27 . J Cell Sci 1997;110:357-68

Hart RP, Rose CS, Hamer RM. Neuropsychological effects of occupational exposure to cadmium. J Clin Exp Neuropsychol 1989;11:933-43

Hashimoto M, Inoue S, Ogawa S, Conrad C, Muramatsu M, Shackelford D, Masliah E. Rapid fragmentation of vimentin in human skin fibroblasts exposed to tamoxifen: a possible involvement of caspase-3. Biochem Biophys Res Commun 1998;247:401-6

Hernandez MR. The optic nerve head in glaucoma: role of astrocytes in tissue remodeling. Prog Retin Eye Res 2000; 19:297-321

Jakob U, Gaestel M, Engel K, Buchner J. Small heat shock proteins are molecular chaperones. J Biol Chem 1993; 268:1517-20

Lee JS, Seo JS. Differential expression of two stress- 
inducible hsp70 genes by various stressors. Exp Mol Med 2002;34:131-6

Lee MW, Park SC, Chae HS, Bach JH, Lee HJ, Lee SH, Kang YK, Kim KY, Lee WB, Kim SS. The protective role of HSP90 against 3-hydroxykynurenine-induced neuronal apoptosis. Biochem Biophys Res Commun 2001;284:261-7

Li CY, Lee JS, Ko YG, Kim JI, Seo JS. Heat shock protein 70 inhibits apoptosis downstream of cytochrome $c$ release and upstream of caspase-3 activation. J Biol Chem 2000a; 275:25665-71

Li M, Kondo T, Zhao QL, Li FJ, Tanabe K, Arai Y, Zhou ZC, Kasuya M. Apoptosis induced by cadmium in human lymphoma U937 cells through Ca2+-calpain and caspase-mitochondria-dependent pathways. J Biol Chem 2000b;275: 39702-9

Mao H, Wang Y, Li Z, Ruchalski KL, Yu X, Schwartz JH, Borkan SC. Hsp72 interacts with paxillin and facilitates the reassembly of focal adhesions during recovery from ATP depletion. J Biol Chem 2004;279:15472-80

Morimoto RI, Tissieres A, Georgopoulos C. The stress response, function of proteins, and oersoectives. In Stress Proteins in Biology and Medicine (Morimoto RI, Tissieres A, Georgopoulos D, eds.), 1990;1-36, Cold Spring Harbor Laboratory, New York, NY

Morishima N. Changes in nuclear morphology during apoptosis correlate with vimentin cleavage by different caspases located either upstream or downstream of Bcl-2 action. Genes Cells 1999:4:401-14

Owen PJ, Johnson GD, Lord JM. Protein kinase C-delta associates with vimentin intermediate filaments in differentiated HL60 cells. Exp Cell Res 1996;225:366-73

Park HS, Lee JS, Huh SH, Seo JS, Choi EJ. Hsp72 Functions as a natural inhibitory protein of c-Jun N-terminal kinase. EMBO J 2001;20:446-56

Paul C, Manero F, Gonin S, Kretz-Remy C, Virot S, Arrigo AP. Hsp27 as a negative regulator of cytochrome $c$ release. Mol Cell Biol 2002;22:816-34

Perng MD, Cairns L, Cairns L, van Den ljssel P, Prescott A, Hutcheson AM, Quinlan RA. Intermediate filament interactions can be altered by HSP27 and alphaB-crystallin. J Cell Sci 1999;112:2099-112

Prasad SC, Thraves PJ, Kuettel MR, Srinivasarao GY, Dritschilo A, Soldatenkov VA. Apoptosis-associated proteolysis of vimentin in human prostate epithelial tumor cells. Biochem Biophys Res Commun 1998;249:332-8
Salvador-Silva M, Ricard CS, Agapova OA, Yang P, Hernandez MR. Expression of small heat shock proteins and intermediate filaments in the human optic nerve head astrocytes exposed to elevated hydrostatic pressure in vitro. $J$ Neurosci Res 2001;66:59-73

Shih CM, Ko WC, Wu JS, Wei YH, Wang LF, Chang EE, Lo TY, Cheng HH, Chen CT. Mediating of caspase-independent apoptosis by cadmium through the mitochondria-ROS pathway in MRC-5 fibroblasts. J Cell Biochem 2004;91: 384-97

Shih CM, Wu JS, Ko WC, Wang LF, Wei YH, Liang HF, Chen YC, Chen CT. Mitochondria-mediated caspase-independent apoptosis induced by cadmium in normal human lung cells. J Cell Biochem 2003;89:335-47

Söllner P, Quinlan RA, Franke WW. Identification of a distinct soluble subunit of an intermediate filament protein: tetrameric vimentin from living cells. Proc Natl Acad Sci USA 1985;82:7929-33

Vicart P, Caron A, Guicheney P, Li Z, Prevost MC, Faure A, Chateau D, Chapon F, Tome F, Dupret JM, Paulin D, Fardeau M. A missense mutation in the alphaB-crystallin chaperone gene causes a desmin-related myopathy. Nat Genet 1998;20:92-5

Wang Z, Templeton DM. Induction of c-fos proto-oncogene in mesangial cells by cadmium. J Biol Chem 1998;273:73-9

Welch WJ. Mammalian stress response: cell physiology, structure/function of stress proteins, and implications for mediate and disease. Physiol Rev 1992;72:1063-81

Wojcik C. Regulation of apoptosis by the ubiquitin and proteasome pathway. J Cell Mol Med 2002;6:25-48

Wu W, Welsh MJ. Expression of the 25-kDa heat-shock protein (HSP27) correlates with resistance to the toxicity of cadmium chloride, mercuric chloride, cis-platinum (II)-diammine dichloride, or sodium arsenite in mouse embryonic stem cells transfected with sense or antisense HSP27 cDNA. Toxicol Appl Pharmacol 1996;141:330-9

Yaglom J, O'Callaghan-Sunol C, Gabai V, Sherman MY. Inactivation of Dual-specificity phosphatases is involved in the regulation of extracellular signal-regulated kinases by heat shock and Hsp72. Mol Cell Biol 2003;23:3813-24

Yoshida S. Re-evaluation of acute neurotoxic effects of $\mathrm{Cd}^{2+}$ on mesencephalic trigeminal neurons of the adult rat. Brain Res 2001;892:102-10 